\title{
Existence Theorems for Quasivariational Inequality Problem on Proximally Smooth Sets
}

\author{
Jittiporn Suwannawit and Narin Petrot \\ Department of Mathematics, Faculty of Science, Naresuan University, Phitsanulok 65000, Thailand \\ Correspondence should be addressed to Narin Petrot; narinp@nu.ac.th
}

Received 1 November 2012; Accepted 23 December 2012

Academic Editor: Pavel Kurasov

Copyright ( 2013 J. Suwannawit and N. Petrot. This is an open access article distributed under the Creative Commons Attribution License, which permits unrestricted use, distribution, and reproduction in any medium, provided the original work is properly cited.

The concept of quasivariational inequality problem on proximally smooth sets is studied. Some sufficient conditions for solving the existence of solutions of such a problem are provided; also some interesting cases are discussed. Of course, due to the significance of proximally smooth sets, the results which are presented in this paper improve and extend many important results in the literature.

\section{Introduction and Preliminaries}

Variational inequality theory is a branch of the mathematics which is important, and it was also the inspiration for researchers to find new works, both in terms of mathematics and applications such as in economics, physical, biological, and engineering science, and other applied sciences. In 1973, Bensoussan et al. [1] introduced and studied the concept of the quasivariational inequality, which is a generalized form of the classical variational inequality that introduced by Stampacchia [2]. Later, many researchers proposed and analyzed the concept of the generalized quasivariational inequality; see, for example [3-18]. It is worth mentioning that the quasivariational inequality problem is of interest to study, since in many important problems the considered set also depends upon the solutions explicitly or implicitly. In fact, the concept of the quasi variational inequality has been applied in many fields such as in economics and transportation equilibrium, control and optimization theory, mathematical programming, and game theory.

In the early period of the research, it should be pointed out that almost all the results regarding the existence and iterative schemes for solving those variational inequalities problems are being considered in the convexity setting. This is because they need the convexity assumption for guaranteeing the well definedness of the proposed iterative algorithm which depends on the projection mapping. However, in fact, the convexity assumption may not be required because it may be well defined even if the considered set is nonconvexs (e.g., when the considered set is a closed subset of a finite dimensional space or a compact subset of a Hilbert space, etc.). However, it may be from the practical point of view one may see that the nonconvex problems are more useful than convex case. Consequently, now many researchers are paying attention to many nonconvex cases.

Let $T: H \rightarrow H$ be a mapping and let $C: H \rightarrow \mathscr{C}(H)$ be a set-valued mapping, where $\mathscr{C}(H)$ is a family of all nonempty closed subsets of $H$. In this paper, we are interested in the following problem: find $x^{*} \in C\left(x^{*}\right)$ such that

$$
-T x^{*} \in N_{C\left(x^{*}\right)}^{P}\left(x^{*}\right),
$$

where $N_{C(x)}^{P}(x)$ is denoted for the proximal normal cone of $C(x)$ of $x$. The problem of type (1) was introduced by Bounkhel et al. [19]. In such a paper, they proposed some iterative algorithms for finding a solution of type (1), when the considered mapping is a set-valued mapping, while, in this paper, we will provide sufficient conditions for the existence of a solution of such a problem (1). To do this, let us start by recalling some basic concepts and useful results that will be needed in this work.

Let $H$ be a real Hilbert space equipped with norm $\|\cdot\|$ and inner product $\langle\cdot, \cdot\rangle$. Let $2^{H}$ be denoted for the class of all nonempty subsets of $H$ and $\mathscr{C}(H)$ denoted for the family of all nonempty closed subsets of $H$. 
For each $K \in 2^{H}$, the usual distance function on $H$ to $K$ is denoted by $d(\cdot, K)$, that is:

$$
d(u, K)=\inf _{v \in K}\|u-v\|, \quad \forall u \in H .
$$

Let $K \in 2^{H}$ and $u \in H$. A point $v \in K$ is called the closest point or the projection of $u$ onto $K$ if

$$
d(u, K)=\|u-v\| .
$$

The set of all such closest points is denoted by $\operatorname{Proj}_{K}(u)$, that is:

$$
\operatorname{Proj}_{K}(u)=\{v \in K: d(u, K)=\|u-v\|\} .
$$

Further, for each $x \in K$, the proximal normal cone to $K$ at $x$ is given by

$$
N_{K}^{P}(x)=\left\{z \in H: \exists \rho>0: x \in \operatorname{Proj}_{K}(x+\rho z)\right\} .
$$

The following is called the proximal normal inequality; the proof of this characterization can be found in [20].

Lemma 1 (see [19]). Let $K$ be a closed subset of a Hilbert space $H$. Then

$$
\begin{aligned}
& z \in N_{K}^{P}(x) \Longleftrightarrow \exists \sigma>0 \\
& \quad \text { such that }\langle z, y-x\rangle \leq \sigma\|y-x\|^{2}, \quad \forall y \in K .
\end{aligned}
$$

We recall also [21] that the Clarke normal cone is given by

$$
N(K, x)=\overline{\mathrm{co}}\left[N_{K}^{P}(x)\right],
$$

where $\overline{\mathrm{co}}[S]$ means the closure of the convex hull of $S$. It is clear that one always has $N_{K}^{P}(x) \subset N(K, x)$. The converse is not true in general. Note that $N(K, x)$ is always a closed and convex cone and that $N_{K}^{P}(x)$ is always a convex cone but may be nonclosed (see $[20,21])$.

In 1995, Clarke et al. [22] have introduced and studied a new class of nonconvex sets, which are called proximally smooth sets. This class of proximally smooth sets has played an important part in many nonconvex applications such as optimization, dynamic systems, and differential inclusions. Subsequently, the proximally smooth sets have been proposed by many researchers. In recent years, Bounkhel et al. [19], Cho et al. [23], Moudafi [24], Noor [25], Noor et al. [26], Petrot [27], and Pang et al. [28] have considered both variational inequalities and equilibrium problems in the context of proximally smooth sets. They suggested and analyzed some projection type iterative algorithms by using the prox-regular technique and auxiliary principle technique. Here, we will take the following characterization proved in [20] as the definition of proximally smooth sets. Note that the original definition was given in terms of the differentiability of the distance function (see $[22,29])$.

Definition 2. For a given $r \in(0,+\infty]$, a subset $K$ of $H$ is said to be uniformly prox-regular with respect to $r$, say, uniformly $r$-prox-regular set, if for all $\bar{x} \in K$ and for all $0 \neq z \in N_{K}^{P}(\bar{x})$, one has

$$
\left\langle\frac{z}{\|z\|}, x-\bar{x}\right\rangle \leq \frac{1}{2 r}\|x-\bar{x}\|^{2}, \quad \forall x \in K .
$$

For the case of $r=\infty$, the uniform $r$-prox-regularity $K$ is equivalent to the convexity of $K$ (see [22]). Moreover, it is known that the class of uniformly prox-regular sets is sufficiently large to include the class $p$-convex sets, $C^{1,1}$ submanifolds (possibly with boundary) of $H$, the images under a $C^{1,1}$ diffeomorphism of convex sets, and many other nonconvex sets; see $[20,29]$.

From now on, we will denote $[\mathrm{Cl}(H)]_{r}$ for the class of all uniformly $r$-prox-regular closed subset of $H$, where $r \epsilon$ $(0,+\infty]$ is fixed positive real number. Also, for each $r \in$ $(0,+\infty]$, we write

$$
K_{r}:=\{x \in H ; d(x, K)<r\} .
$$

Remark 3. If $T: H \rightarrow H$ and $C: H \rightarrow[\mathrm{Cl}(H)]_{r}$ are mappings, then the problem of type (1) is equivalent to the following problem: find $x^{*} \in C\left(x^{*}\right)$ such that

$$
\left\langle T x^{*}, x-x^{*}\right\rangle+\frac{\left\|T x^{*}\right\|}{2 r}\left\|x-x^{*}\right\|^{2} \geq 0, \quad \forall x \in C\left(x^{*}\right),
$$

see [19]. This means, in particular, that the problem (1) contains the well-known Stampacchia's variational inequality, as a special case.

The following lemma which summarizes some important consequences of the uniformly prox-regularity sets is needed in the sequel. The proof of this result can be found in $[22,29]$.

Lemma 4. Let $r \in(0,+\infty]$ and $K$ be a nonempty closed subset of $H$. If $K$ is uniformly r-prox-regular set, then the following holds.

(i) For all $x \in K_{r}, \operatorname{Proj}_{K}(x) \neq \emptyset$.

(ii) For all $s \in(0, r), \operatorname{Proj}_{K}$ is Lipschitz with constant $r /(r-$ s) on $K_{s}$.

(iii) The proximal normal cone is closed as a set-valued mapping.

Remark 5. If $K$ is uniformly $r$-prox-regular set, as a direct consequence of Lemma 4 (iii), we have $N(K, x)=N_{K}^{P}(x)$.

The following definition and lemma are also needed, in order to obtain our main results.

Definition 6. A set-valued mapping $C: H \rightarrow 2^{H}$ is said to be $\kappa$-Lipschitz if there exists $\kappa>0$ such that

$$
\begin{array}{r}
\left|d(y, C(x))-d\left(y^{\prime}, C\left(x^{\prime}\right)\right)\right| \leq\left\|y-y^{\prime}\right\|+\kappa\left\|x-x^{\prime}\right\|, \\
\forall x, x^{\prime}, y, y^{\prime} \in H .
\end{array}
$$

Lemma 7 (see [19]). Let $r \in(0,+\infty]$ and let $C: H \rightarrow 2^{H}$ be a $\kappa$-Lipschitz set-valued mapping with uniformly $r$-proxregular values then the following closedness property holds: "For any $x_{n} \rightarrow x^{*}, y_{n} \rightarrow y^{*}$ and $u_{n} \rightarrow u^{*}$ with $y_{n} \in C\left(x_{n}\right)$ and $u_{n} \in N\left(C\left(x_{n}\right), y_{n}\right)$, one has $u^{*} \in N\left(C\left(x^{*}\right), y^{*}\right)$." 


\section{Main Results}

In this paper, we are interested in the following classes of mappings.

Definition 8. Let $T: H \rightarrow H$ be a mapping. Then $T$ is called

(a) $\beta$-strongly monotone if there exists $\beta>0$ such that

$$
\langle T(x)-T(y), x-y\rangle \geq \beta\|x-y\|^{2}, \quad \forall x, y \in H,
$$

(b) $\xi$-Lipschitz if there exists $\xi>0$ such that

$$
\|T(x)-T(y)\| \leq \xi\|x-y\|, \quad \forall x, y \in H .
$$

That is, in other word, we will make the following assumption.

Assumption $(\mathscr{A})$. Let $T: H \rightarrow H$ and $C: H \rightarrow[\mathrm{Cl}(H)]_{r}$ be mappings.

(i) $T$ is a $\beta$-strongly monotone and a $\xi$-Lipschitz singlevalued mapping;

(ii) $C$ is a $\kappa$-Lipschitz set-valued mapping;

(iii) there is $\omega \in[0,1)$ such that

$$
\left\|\operatorname{Proj}_{C(x)}(z)-\operatorname{Proj}_{C(y)}(z)\right\| \leq \omega\|x-y\|, \quad \forall x, y, z \in H .
$$

Remark 9. Let $H=(-\infty, \infty)$ and $K=[a, b] \cup[c, d]$, for some positive real numbers $a, b, c, d$ with $a<b<c<d$. If we define $C: H \rightarrow 2^{H}$ by $C(x)=m(x)+K$, where $m: H \rightarrow H$ is a mapping defined by $m(x)=k x$ for $k \in R$, then we see that $C$ is a $\max \{d-b, c-a\}$-Lipschitz mapping, and Assumption $(\mathscr{A})$ (iii) is satisfied with a constant $2(\max \{d-b, c-a\})$. This means that Assumptions ( $\mathscr{A})$ (ii) and (iii) are independent.

The following remark is very useful in order to prove our results. Before seeing that, for the sake of simplicity, let us make a notation: for each $r \in(0, \infty)$ and $s \in(0, r)$, we will write $t_{s}:=r /(r-s)$.

Remark 10. Let $r, \beta, \xi, \omega$, and $\delta$ be five positive real numbers such that $\beta \in(0, \xi), \delta \in\left(0, r \sqrt{\beta^{2}-\xi^{2}\left(1-(1-\omega)^{2}\right)} /(1-\omega)^{2}\right)$ and $\omega \in\left[0,1-\xi r \sqrt{\xi^{2}-\beta^{2}} /\left(\xi^{2} r-\beta \delta\right)\right)$. If $h:[1, \diamond] \rightarrow[0, \infty)$ is a function defined by

$$
h(t)=\frac{r(t-1)}{t \delta}+\frac{\sqrt{\beta^{2} t^{2}-\xi^{2}\left[t^{2}-(1-\omega)^{2}\right]}}{t \xi^{2}}
$$

for all $t \in[1, \diamond]$, where $\diamond=\xi(1-\omega) / \sqrt{\xi^{2}-\beta^{2}}$, then $h$ is an increasing continuous function on its domain. Moreover, we can check that $\beta / \xi^{2}$ is an element of the range of $h$.

Next, for a fixed positive real number $\kappa \in[0,1 /(2 \diamond-1))$, we pick a real number $\zeta=(1-\diamond \kappa) / \kappa(\diamond-1)$. Here, we notice that $\zeta \in(1, \infty)$. Let us consider now a case when $h^{-1}\left(\beta / \xi^{2}\right) \epsilon$ $[1,(1+\zeta \kappa) / \kappa(1+\zeta)]$. Then, by the definition of $t_{s}$ and $h$ is an increasing continuous function, for $s^{*}=r(1-\kappa) /(1+\zeta \kappa)$ we have

$$
\frac{\beta}{\xi^{2}}<\frac{r\left(t_{s^{*}}-1\right)}{t_{s^{*}} \delta}+\frac{\sqrt{\beta^{2} t_{s^{*}}^{2}-\xi^{2}\left[t_{s^{*}}^{2}-(1-\omega)^{2}\right]}}{t_{s^{*}} \xi^{2}},
$$

that is,

$$
\frac{\beta}{\xi^{2}}-\frac{\sqrt{\beta^{2} t_{s^{*}}^{2}-\xi^{2}\left[t_{s^{*}}^{2}-(1-\omega)^{2}\right]}}{t_{s^{*}} \xi^{2}}<\frac{r\left(t_{s^{*}}-1\right)}{t_{s^{*}} \delta} .
$$

Since $r\left(t_{s^{*}}-1\right) / t_{s^{*}}=s^{*}$, this gives

$$
\frac{\beta}{\xi^{2}}-\frac{\sqrt{\beta^{2} t_{s^{*}}^{2}-\xi^{2}\left[t_{s^{*}}^{2}-(1-\omega)^{2}\right]}}{t_{s^{*}} \xi^{2}}<\frac{s^{*}}{\delta} .
$$

This allows us to take a real number $\rho$ such that

$$
\frac{\beta}{\xi^{2}}-f\left(t_{s^{*}}\right)<\rho<\min \left\{\frac{\beta}{\xi^{2}}+f\left(t_{s^{*}}\right), \frac{s^{*}}{\delta}\right\},
$$

where $f(t)=\sqrt{\beta^{2} t^{2}-\xi^{2}\left[t^{2}-(1-\omega)^{2}\right]} / t \xi^{2}$.

Now we are in a position to present our main results.

Theorem 11. Let $T: H \rightarrow H$ be a single-valued mapping and let $\mathrm{C}: H \rightarrow[\mathrm{Cl}(H)]_{r}$ be a set-valued mapping. Assume that Assumption $(\mathscr{A})$ holds and the following control conditions are satisfied:

(i) $\delta \in\left(0, r \sqrt{\beta^{2}-\xi^{2}\left(1-(1-\omega)^{2}\right)} /(1-\omega)^{2}\right)$ and $\omega \epsilon$ $\left[0,1-\xi r \sqrt{\xi^{2}-\beta^{2}} /\left(\xi^{2} r-\beta \delta\right)\right)$, where $\delta=\sup \{\|T(x)\|:$ $x \in H\}$;

(ii) $\kappa \in[0,1 /(2 \diamond-1))$, where $\diamond=\xi(1-\omega) / \sqrt{\xi^{2}-\beta^{2}}$;

(iii) $h^{-1}\left(\beta / \xi^{2}\right) \in[1,(1+\zeta \kappa) / \kappa(1+\zeta)]$, where $h$ and $\zeta$ are defined as Remark 10.

If there is $x_{0} \in H$ such that $d\left(x_{0}, C\left(x_{0}\right)\right)<s^{*}-\rho\left\|T x_{0}\right\|$, where $s^{*}=r(1-\kappa) /(1+\zeta \kappa)$ and $\rho$ is a real number corresponding to ${ }^{*}$ which is chosen as in (19), then the problem (1) has a solution.

Proof. Firstly, we will define a sequence $\left\{x_{n}\right\}$ in $H$ as follows: consider an element $x_{0}$ in $H$ such that $d\left(x_{0}, C\left(x_{0}\right)\right)<s^{*}-$ $\rho\left\|T x_{0}\right\|$; we see that

$$
d\left(x_{0}-\rho T x_{0}, C\left(x_{0}\right)\right) \leq d\left(x_{0}, C\left(x_{0}\right)\right)+\rho\left\|T x_{0}\right\|<s^{*} \text {. }
$$


This means $x_{0}-\rho T x_{0} \in\left[C\left(x_{0}\right)\right]_{s^{*}}$. Subsequently, by Lemma 4 (i), we know that $\operatorname{Proj}_{C\left(x_{0}\right)}\left[x_{0}-\rho T x_{0}\right] \neq \emptyset$. Let $x_{1} \in$ $\operatorname{Proj}_{C\left(x_{0}\right)}\left[x_{0}-\rho T x_{0}\right]$. So, by a choice of $\rho$, we see that

$$
\begin{aligned}
d\left(x_{1}\right. & \left.-\rho T x_{1}, C\left(x_{1}\right)\right) \\
& \leq d\left(x_{1}, C\left(x_{1}\right)\right)+\rho\left\|T x_{1}\right\| \\
& =d\left(x_{1}, C\left(x_{1}\right)\right)-d\left(x_{1}, C\left(x_{0}\right)\right)+\rho\left\|T x_{1}\right\| \\
& \leq \kappa\left\|x_{1}-x_{0}\right\|+\rho\left\|T x_{1}\right\| \\
& <s^{*}+\kappa\left\|x_{1}-x_{0}\right\| .
\end{aligned}
$$

On the other hand, we see that

$$
\begin{aligned}
\| x_{1}- & x_{0} \| \\
& \leq\left\|x_{1}-\left(x_{0}-\rho T x_{0}\right)\right\|+\left\|\left(x_{0}-\rho T x_{0}\right)-x_{0}\right\| \\
& =d\left(x_{0}-\rho T x_{0}, C\left(x_{0}\right)\right)+\rho\left\|T x_{0}\right\| \\
& \leq d\left(x_{0}, C\left(x_{0}\right)\right)+\rho\left\|T x_{0}\right\|+\rho\left\|T x_{0}\right\| \\
& <\left(s^{*}-\rho\left\|T x_{0}\right\|\right)+\rho\left\|T x_{0}\right\|+\rho\left\|T x_{0}\right\| \\
& <2 s^{*} .
\end{aligned}
$$

Using this one together with (21), we obtain

$$
d\left(x_{1}-\rho T x_{1}, C\left(x_{1}\right)\right)<s^{*}+2 \kappa s^{*} \text {. }
$$

Note that, by $\zeta>1$, we have $(1-\kappa)(1+2 \kappa) /(1+\zeta \kappa)<1$. So, since $s^{*}=r(1-\kappa) /(1+\zeta \kappa)$, by $(23)$ we have

$$
\begin{aligned}
d\left(x_{1}-\rho T x_{1}, C\left(x_{1}\right)\right) & <\frac{r(1-\kappa)(1+2 \kappa)}{1+\zeta \kappa} \\
& <r .
\end{aligned}
$$

Hence $x_{1}-\rho T x_{1} \in\left[C\left(x_{1}\right)\right]_{r(1-\kappa)(1+2 \kappa) /(1+\zeta \kappa)}$, and it follows that $\operatorname{Proj}_{C\left(x_{1}\right)}\left[x_{1}-\rho T x_{1}\right] \neq \emptyset$. Let $x_{2} \in \operatorname{Proj}_{C\left(x_{1}\right)}\left[x_{1}-\rho T x_{1}\right]$. In the same way as obtaining (21) and (23), we see that

$$
\begin{aligned}
& d\left(x_{2}-\rho T x_{2}, C\left(x_{2}\right)\right)<s^{*}+\kappa\left\|x_{2}-x_{1}\right\|, \\
& \left\|x_{2}-x_{1}\right\| \\
& \quad \leq d\left(x_{1}, C\left(x_{1}\right)\right)+2 \rho\left\|T x_{1}\right\| \\
& \quad=d\left(x_{1}, C\left(x_{1}\right)\right)-d\left(x_{1}, C\left(x_{0}\right)\right)+2 \rho\left\|T x_{1}\right\| \\
& \quad \leq \kappa\left\|x_{1}-x_{0}\right\|+2 \rho\left\|T x_{1}\right\| \\
& \quad=2 s^{*}+\kappa\left\|x_{1}-x_{0}\right\| \\
& <2 s^{*}+2 \kappa s^{*} .
\end{aligned}
$$

By (25), we have

$$
\begin{aligned}
d\left(x_{2}-\rho T x_{2}, C\left(x_{2}\right)\right) & <s^{*}+\kappa\left(2 \kappa s^{*}+2 s^{*}\right) \\
& =s^{*}+2 \kappa s^{*}+2 \kappa^{2} s^{*} \\
& \leq r\left(\frac{1-\kappa}{1+\zeta \kappa}\right)\left(1+2 \kappa+2 \kappa^{2}+\cdots\right) \\
& =r\left(\frac{1-\kappa}{1+\zeta \kappa}\right)\left(\frac{1+\kappa}{1-\kappa}\right) \\
& <r .
\end{aligned}
$$

By continuing this process, we can construct a sequence $\left\{x_{n}\right\}$ in $H$ such that

$$
\begin{gathered}
x_{n+1} \in \operatorname{Proj}_{C\left(x_{n}\right)}\left[x_{n}-\rho T x_{n}\right], \\
d\left(x_{n}-\rho T x_{n}, C\left(x_{n}\right)\right)<s^{*}+\kappa\left\|x_{n}-x_{n-1}\right\|,
\end{gathered}
$$

for all $n \in \mathbb{N}$.

Further, let us consider

$$
\begin{aligned}
\left\|x_{n}-x_{n-1}\right\| \leq & \left\|x_{n}-\left(x_{n-1}-\rho T x_{n-1}\right)\right\| \\
& +\left\|\left(x_{n-1}-\rho T x_{n-1}\right)-x_{n-1}\right\| \\
= & d\left(x_{n-1}-\rho T x_{n-1}, C\left(x_{n-1}\right)\right)+\rho\left\|T x_{n-1}\right\| \\
< & s^{*}+\kappa\left\|x_{n-1}-x_{n-2}\right\|+\rho\left\|T x_{n-1}\right\| \\
= & 2 s^{*}+\kappa\left\|x_{n-1}-x_{n-2}\right\| \\
< & 2 s^{*}+2 \kappa s^{*}+\cdots+2 \kappa^{n-2}+2 \kappa^{n-1} s^{*} \\
= & 2 s^{*}\left(1+\kappa+\cdots+\kappa^{n-2}+\kappa^{n-1}\right) \\
= & 2 s^{*}\left(\frac{1-\kappa^{n}}{1-\kappa}\right),
\end{aligned}
$$

for each $n \in \mathbb{N}$. This implies

$$
\begin{aligned}
d\left(x_{n}-\rho T x_{n}, C\left(x_{n}\right)\right) & <s^{*}+2 \kappa s^{*}\left(\frac{1-\kappa^{n}}{1-\kappa}\right) \\
& =s^{*}\left(1+2 \kappa\left(\frac{1-\kappa^{n}}{1-\kappa}\right)\right) \\
& =s^{*}\left(\frac{1-\kappa+2 \kappa-2 \kappa^{n+1}}{1-\kappa}\right) \\
& \leq s^{*}\left(\frac{1+\kappa}{1-\kappa}\right) \\
& <r\left(\frac{1+\kappa}{1+\zeta \kappa}\right) \\
& <r .
\end{aligned}
$$


Write $\lambda:=r((1+\kappa) /(1+\zeta \kappa))$. From the previous argument, we see that the sequence $\left\{x_{n}\right\}$ also has a property that

$$
x_{n}-\rho T x_{n} \in\left[C\left(x_{n}\right)\right]_{\lambda},
$$

for all $n \in \mathbb{N}$.

Next, we will show that $\left\{x_{n}\right\}$ is a convergent sequence, and its limit is nothing but a solution of the problem (1).

Now, by using the Assumption (A) (iii) and Lemma 4 (ii), we have

$$
\begin{aligned}
& \left\|x_{n+1}-x_{n}\right\| \\
& =\left\|\operatorname{Proj}_{C\left(x_{n}\right)}\left(x_{n}-\rho T x_{n}\right)-\operatorname{Proj}_{C\left(x_{n-1}\right)}\left(x_{n-1}-\rho T x_{n-1}\right)\right\| \\
& \leq\left\|\operatorname{Proj}_{C\left(x_{n}\right)}\left(x_{n}-\rho T x_{n}\right)-\operatorname{Proj}_{C\left(x_{n}\right)}\left(x_{n-1}-\rho T x_{n-1}\right)\right\| \\
& \quad+\| \operatorname{Proj}_{C\left(x_{n}\right)}\left(x_{n-1}-\rho T x_{n-1}\right) \\
& \quad-\operatorname{Proj}_{C\left(x_{n-1}\right)}\left(x_{n-1}-\rho T x_{n-1}\right) \| \\
& \leq t_{\lambda}\left\|x_{n}-\rho T x_{n}-x_{n-1}+\rho T x_{n-1}\right\|+\omega\left\|x_{n}-x_{n-1}\right\| .
\end{aligned}
$$

Meanwhile, since $T$ is a $\beta$-strongly monotone and $\xi$-Lipschitz mapping, we see that

$$
\begin{aligned}
\| x_{n}- & \rho T x_{n}-x_{n-1}+\rho T x_{n-1} \|^{2} \\
= & \left\|x_{n}-x_{n-1}-\rho\left[T x_{n}-T x_{n-1}\right]\right\|^{2} \\
= & \left\|x_{n}-x_{n-1}\right\|^{2}-2 \rho\left\langle T x_{n}-T x_{n-1}, x_{n}-x_{n-1}\right\rangle \\
& +\rho^{2}\left\|T x_{n}-T x_{n-1}\right\|^{2} \\
\leq & \left\|x_{n}-x_{n-1}\right\|^{2}-2 \rho \beta\left\|x_{n}-x_{n-1}\right\|^{2} \\
& +\rho^{2} \xi^{2}\left\|x_{n}-x_{n-1}\right\|^{2} \\
= & \left(1-2 \rho \beta+\rho^{2} \xi^{2}\right)\left\|x_{n}-x_{n-1}\right\|^{2} .
\end{aligned}
$$

Let $\theta_{\rho}=\max \left\{1-2 \rho \beta+\rho^{2} \xi^{2},((1-\omega)(r-\lambda) / 2 r)^{2}\right\}$. Observe that $\theta_{\rho} \in[0, \infty)$. Moreover, it follows that

$$
\left\|x_{n}-\rho T x_{n}-x_{n-1}+\rho T x_{n-1}\right\| \leq \sqrt{\theta_{\rho}}\left\|x_{n}-x_{n-1}\right\|
$$

By replacing (33) into (31), we get

$$
\begin{aligned}
\left\|x_{n+1}-x_{n}\right\| & \leq t_{\lambda} \sqrt{\theta_{\rho}}\left\|x_{n}-x_{n-1}\right\|+\omega\left\|x_{n}-x_{n-1}\right\| \\
& =\left[t_{\lambda} \sqrt{\theta_{\rho}}+\omega\right]\left\|x_{n}-x_{n-1}\right\| .
\end{aligned}
$$

Let $\gamma:=t_{\lambda} \sqrt{\theta_{\rho}}+\omega$. Then, by a choice of $\rho$, one can check that $\gamma<1$. Subsequently, by (34), we have

$$
\left\|x_{n+1}-x_{n}\right\| \leq \gamma^{n}\left\|x_{1}-x_{0}\right\|, \quad \forall n \in \mathbb{N} \text {. }
$$

Hence, for any $m \geq n$, we see that

$$
\begin{aligned}
\left\|x_{m}-x_{n}\right\| & \leq \sum_{i=n}^{m-1}\left\|x_{i+1}-x_{i}\right\| \\
& \leq\left\|x_{1}-x_{0}\right\| \sum_{i=n}^{m-1} \gamma^{i} \\
& \leq\left\|x_{1}-x_{0}\right\| \sum_{i=n}^{\infty} \gamma^{i} \\
& \leq\left(\frac{\gamma^{n}}{1-\gamma}\right)\left\|x_{1}-x_{0}\right\| .
\end{aligned}
$$

This implies that $\left\{x_{n}\right\}$ is a Cauchy sequence in $H$, since $\gamma<1$. So, by the completeness of $H$, there exists $x^{*} \in H$ such that $x_{n} \rightarrow x^{*}$ as $n \rightarrow \infty$.

We now finish the proof by showing that $x^{*}$ is a solution of problem (1). To do this, we will start by asserting that $x^{*} \in C\left(x^{*}\right)$. Indeed, since $x_{n+1} \in C\left(x_{n}\right)$ and $C$ is a $\kappa$-Lipschitz mapping, we have

$$
\begin{aligned}
d\left(x^{*}, C\left(x^{*}\right)\right) & =\left|d\left(x_{n+1}, C\left(x_{n}\right)\right)-d\left(x^{*}, C\left(x^{*}\right)\right)\right| \\
& \leq\left\|x_{n+1}-x^{*}\right\|+\kappa\left\|x_{n}-x^{*}\right\| .
\end{aligned}
$$

Thus, since $x_{n} \rightarrow x^{*}$, we see that $d\left(x^{*}, C\left(x^{*}\right)\right)=0$. So, by the closedness of $C\left(x^{*}\right)$, it guarantees that $x^{*} \in C\left(x^{*}\right)$.

Next we show that $T x^{*} \in-N\left(C\left(x^{*}\right), x^{*}\right)$. Let us observe that

$$
x_{n+1} \in \operatorname{Proj}_{C\left(x_{n}\right)}\left(x_{n}-\rho T x_{n}\right), \quad \forall n \in \mathbb{N}
$$

is equivalent to

$$
x_{n+1} \in \operatorname{Proj}_{C\left(x_{n}\right)}\left(x_{n+1}+\left(x_{n}-x_{n+1}\right)-\rho T x_{n}\right) .
$$

And this means

$$
\left(x_{n}-x_{n+1}\right)-\rho T x_{n} \in N\left(C\left(x_{n}\right), x_{n+1}\right), \quad \forall n \in \mathbb{N} .
$$

Thus, by using the continuity of mapping $T$, from Lemma 7 we see that $\rho T x^{*} \in-N\left(C\left(x^{*}\right), x^{*}\right)$. This implies $T x^{*} \in$ $-N\left(C\left(x^{*}\right), x^{*}\right)$, as required. This means that $x^{*}$ is a solution of the problem (1), and the proof is completed.

Remark 12. A condition which has been proposed in the assumptions of Theorem 11 is that "there is $x_{0} \in H$ such that $d\left(x_{0}, C\left(x_{0}\right)\right)<s^{*}-\rho\left\|T x_{0}\right\|$, where $s^{*}=r(1-\kappa) /(1+\zeta \kappa)$." Here, in view of Remark 10 together with the following facts, one may see that our choice $s^{*}=r(1-\kappa) /(1+\zeta \kappa)$ should be sharpest.

(i) $s=r(1-\kappa) /(1+\zeta \kappa) \Leftrightarrow t_{s}=(1+\zeta \kappa) / \kappa(1+\zeta)$.

(ii) The function $\zeta \mapsto(1+\zeta \kappa) / \kappa(1+\zeta)$ is an increasing function on its domain.

(iii) $(1+\zeta \kappa) / \kappa(1+t)=\diamond \Leftrightarrow \zeta=(1-\diamond \kappa) / \kappa(\diamond-1)$, where $\diamond=\xi(1-\omega) / \sqrt{\xi^{2}-\beta^{2}}$. 
Remark 13. Assume that all assumptions of Theorem 11 hold. By starting with an element $x_{0} \in H$ such that $d\left(x_{0}, C\left(x_{0}\right)\right)<$ $s^{*}-\rho\left\|T x_{0}\right\|$, a sequence $\left\{x_{n}\right\} \subset H$ which is defined by

$$
x_{n+1} \in \operatorname{Proj}_{C\left(x_{n}\right)}\left[x_{n}-\rho T x_{n}\right]
$$

is well defined. Moreover, it is a convergent sequence and its limit is a solution of the problem (1).

Remark 14. Recall that a set-valued mapping $C: H \rightarrow 2^{H}$ is said to be Hausdorff Lipschitz if there exists a real number $\kappa>0$ such that

$$
\mathscr{H}(C(x), C(y)) \leq \kappa\|x-y\|, \quad \forall x, y \in H,
$$

where $\mathscr{H}$ stands for the Hausdorff distance relative to the norm associated with the Hilbert space $H$, that is:

$$
\begin{array}{r}
\mathscr{H}(A, B)=\max \left\{\sup _{x \in A} \inf _{y \in B}\|x-y\|, \sup _{y \in B} \inf _{x \in A}\|x-y\|\right\}, \\
\forall A, B \in 2^{H} .
\end{array}
$$

It is easy to check that the class of Lipschitz mappings, which has been defined in Definition 6, is larger than the class of Hausdorff Lipschitz mappings. Thus, Theorem 11 can also be applied when the Assumption $(\mathscr{A})$ (ii) is replaced by " $C$ is $\kappa$ Hausdorff Lipschitz set-valued mapping."

Remark 15 . Let $K$ be a uniformly prox-regular closed subset of $H$. If $C: H \rightarrow[\mathrm{Cl}(H)]_{r}$ is defined by

$$
C(x)=K, \quad \forall x \in H,
$$

then we see that Assumptions (A) (ii) and (iii) are satisfied with a constant zero. In this case, Theorem 11 is reduced to a result which was presented by Petrot [27].

It is well known that if $K$ is a closed convex set, then it is $r$-prox-regular set for every $r>0$ (see [22]). Using this fact, and by careful consideration of the proof of Theorem 11, one can see that control conditions (i) and (iii) of Theorem 11 can be omitted. So, we have the following results immediately.

Corollary 16. Let $T: H \rightarrow H$ be a single-valued mapping and let $C: H \rightarrow \mathscr{C} \mathscr{C}(H)$ be a set-valued mapping, where $\mathscr{C} \mathscr{C}(H)$ is a family of nonempty closed convex subset of $H$. If the Assumption $(\mathscr{A})$ holds and $\kappa \in[0,1 /(2 \diamond-1))$, where $\diamond=$ $\xi(1-\omega) / \sqrt{\xi^{2}-\beta^{2}}$, then the problem (1) has a solution.

Finally, in view of Remark 15 and Corollary 16, we can obtain the following classical result immediately.

Corollary 17. Let $H$ be a real Hilbert space, let $K$ be a closed convex subset of $H$, and let $T: H \rightarrow H$ be a single-valued mapping. If $T$ is a strongly monotone and Lipschitz singlevalued mapping, then the problem (1) has a solution.

\section{Conclusion}

In this paper, we provide some conditions for the existence theorems of the quasivariational inequality problem on a class of nonconvex sets. In fact, there are two constraints on the assumptions of considered mapping $T$ in the main Theorem 11, that is,

(i) $T$ is a strongly monotone and Lipschitz mapping,

(ii) the range of mapping $T$ is a bounded set.

Hence, these lead to some natural questions in the future works for relaxing these constraints. At this point, we desire that the results presented here will be useful for those researchers, because this paper may also be extended and generalized for considering the mutlivalued and set-valued extended general variational inequalities problems.

\section{Acknowledgment}

This work is supported by the Centre of Excellence in Mathematics, Commission on Higher Education, Thailand.

\section{References}

[1] A. Bensoussan, M. Goursat, and J.-L. Lions, "Contrôle impulsionnel et inéquations quasi-variationnelles stationnaires," vol. 276, pp. A1279-A1284, 1973.

[2] G. Stampacchia, "Formes bilinéaires coercitives sur les ensembles convexes," vol. 258, pp. 4413-4416, 1964.

[3] M. Alimohammady, J. Balooee, Y. J. Cho, and M. Roohi, "Iterative algorithms for a new class of extended general nonconvex set-valued variational inequalities," Nonlinear Analysis: Theory, Methods \& Applications A, vol. 73, no. 12, pp. 3907-3923, 2010.

[4] J. Balooee and Y. J. Cho, "Algorithms for solutions of extended general mixed variational inequalities and fixed points," Optimization Letters, p. 27, 2012.

[5] S. S. Chang, Variational Inequality and Complementarity Problem Theory with Applications, Shanghai Scientific and Technology Literature, Shanghai, China, 1991.

[6] W. Chantarangsi, C. Jaiboon, and P. Kumam, "A viscosity hybrid steepest descent method for generalized mixed equilibrium problems and variational inequalities for relaxed cocoercive mapping in Hilbert spaces," Abstract and Applied Analysis, vol. 2010, Article ID 390972, 39 pages, 2010.

[7] S.-S. Chang, B. S. Lee, X. Wu, Y. J. Cho, and G. M. Lee, "On the generalized quasi-variational inequality problems," Journal of Mathematical Analysis and Applications, vol. 203, no. 3, pp. 686-711, 1996.

[8] Y. J. Cho and X. Qin, "Systems of generalized nonlinear variational inequalities and its projection methods," Nonlinear Analysis: Theory, Methods \& Applications A, vol. 69, no. 12, pp. 4443-4451, 2008.

[9] G. Gu, S. Wang, and Y. J. Cho, "Strong convergence algorithms for hierarchical fixed points problems and variational inequalities," Journal of Applied Mathematics, vol. 2011, Article ID 164978, 17 pages, 2011.

[10] N.-J. Huang, M.-R. Bai, Y. J. Cho, and S. M. Kang, "Generalized nonlinear mixed quasi-variational inequalities," Computers \& Mathematics with Applications, vol. 40, no. 2-3, pp. 205-215, 2000.

[11] N. J. Huang, "On the generalized implicit quasivariational inequalities," Journal of Mathematical Analysis and Applications, vol. 216, no. 1, pp. 197-210, 1997. 
[12] C. Jaiboon and P. Kumam, "A general iterative method for solving equilibrium problems, variational inequality problems and fixed point problems of an infinite family of nonexpansive mappings," Journal of Applied Mathematics and Computing, vol. 34, no. 1-2, pp. 407-439, 2010.

[13] P. Kumam, "Strong convergence theorems by an extragradient method for solving variational inequalities and equilibrium problems in a Hilbert space," Turkish Journal of Mathematics, vol. 33, no. 1, pp. 85-98, 2009.

[14] X. Qin, M. Shang, and Y. Su, "Strong convergence of a general iterative algorithm for equilibrium problems and variational inequality problems," Mathematical and Computer Modelling, vol. 48, no. 7-8, pp. 1033-1046, 2008.

[15] X. Qin, M. Shang, and H. Zhou, "Strong convergence of a general iterative method for variational inequality problems and fixed point problems in Hilbert spaces," Applied Mathematics and Computation, vol. 200, no. 1, pp. 242-253, 2008.

[16] S. Suantai and N. Petrot, "Existence and stability of iterative algorithms for the system of nonlinear quasi-mixed equilibrium problems," Applied Mathematics Letters, vol. 24, no. 3, pp. 308313, 2011.

[17] Y. H. Yao, Y. C. Liou, and J. C. Yao, "An extra gradient method for fixed point problems and variational inequality problems," Journal of Inequalities and Applications, vol. 2007, Article ID 38752, 12 pages, 2007.

[18] Y. Yao, Y.-C. Liou, and J.-C. Yao, "A new hybrid iterative algorithm for fixed-point problems, variational inequality problems, and mixed equilibrium problems," Fixed Point Theory and Applications, vol. 2008, Article ID 417089, 15 pages, 2008.

[19] M. Bounkhel, L. Tadj, and A. Hamdi, "Iterative schemes to solve nonconvex variational problems," Journal of Inequalities in Pure and Applied Mathematics, vol. 4, no. 1, 14 pages, 2003.

[20] F. H. Clarke, Yu. S. Ledyaev, R. J. Stern, and P. R. Wolenski, Nonsmooth Analysis and Control Theory, vol. 178, Springer, New York, NY, USA, 1998.

[21] F. H. Clarke, Optimization and Nonsmooth Analysis, WileyInterscience, New York, NY, USA, 1983.

[22] F. H. Clarke, R. J. Stern, and P. R. Wolenski, "Proximal smoothness and the lower $C^{2}$ property," Journal of Convex Analysis, vol. 2, no. 1-2, pp. 117-144, 1995.

[23] Y. J. Cho, J. K. Kim, and R. U. Verma, "A class of nonlinear variational inequalities involving partially relaxed monotone mappings and general auxiliary problem principle," Dynamic Systems and Applications, vol. 11, no. 3, pp. 333-338, 2002.

[24] A. Moudafi, "An algorithmic approach to prox-regular variational inequalities," Applied Mathematics and Computation, vol. 155, no. 3, pp. 845-852, 2004.

[25] M. A. Noor, "Iterative schemes for nonconvex variational inequalities," Journal of Optimization Theory and Applications, vol. 121, no. 2, pp. 385-395, 2004.

[26] M. A. Noor, N. Petrot, and J. Suwannawit, "Existence theorems for multivalued variational inequality problems on uniformly prox-regular sets," Optimization Letters, 2012.

[27] N. Petrot, "Some existence theorems for nonconvex variational inequalities problems," Abstract and Applied Analysis, vol. 2010, Article ID 472760, 9 pages, 2010.

[28] L.-P. Pang, J. Shen, and H.-S. Song, "A modified predictorcorrector algorithm for solving nonconvex generalized variational inequality," Computers \& Mathematics with Applications, vol. 54, no. 3, pp. 319-325, 2007.
[29] R. A. Poliquin, R. T. Rockafellar, and L. Thibault, "Local differentiability of distance functions," Transactions of the American Mathematical Society, vol. 352, no. 11, pp. 5231-5249, 2000. 


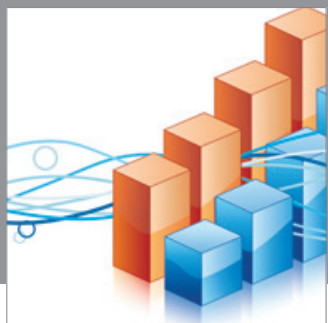

Advances in

Operations Research

mansans

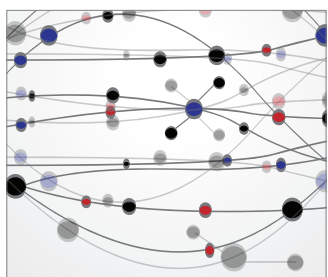

The Scientific World Journal
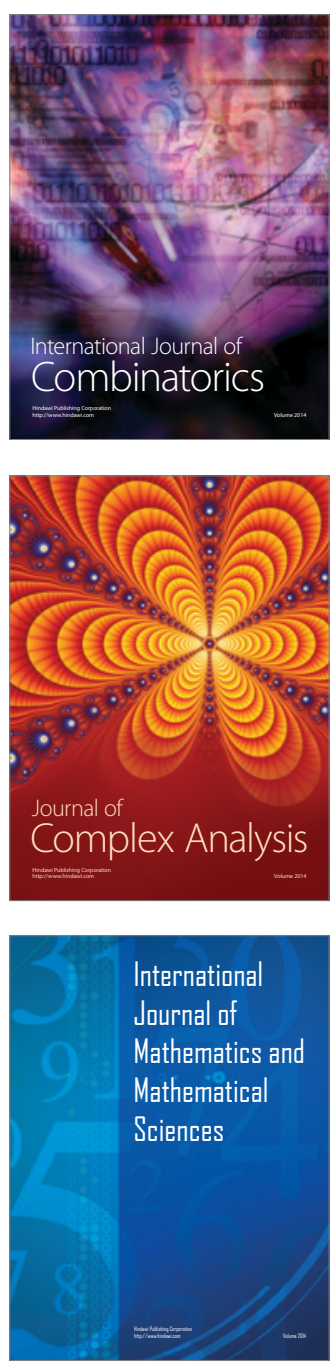
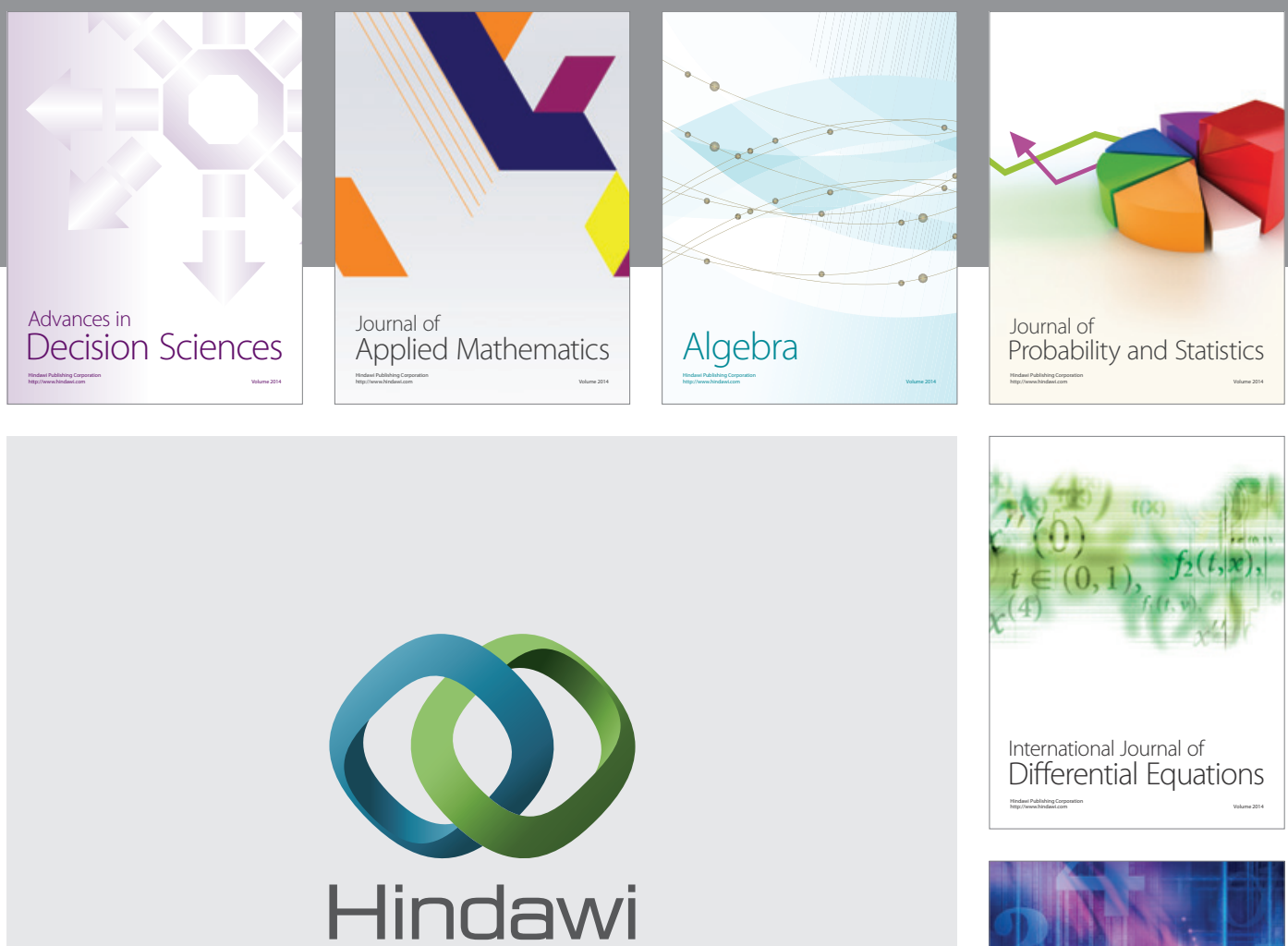

Submit your manuscripts at http://www.hindawi.com
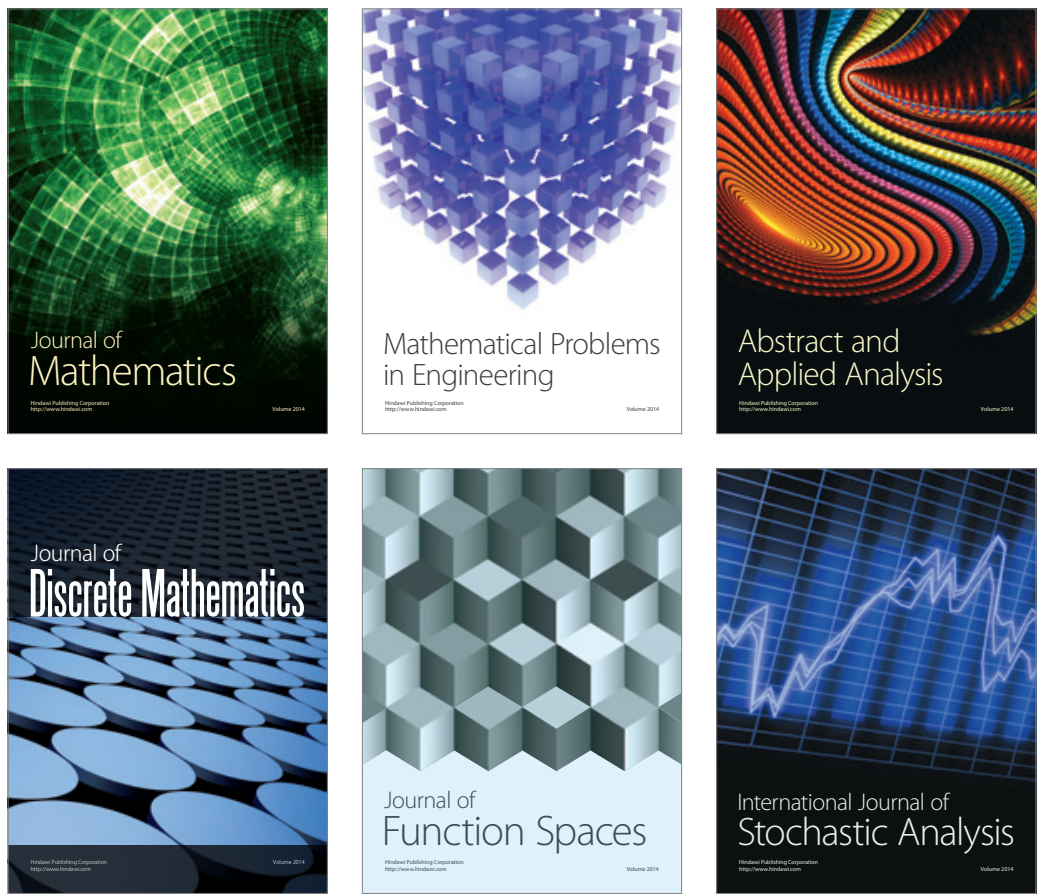

Journal of

Function Spaces

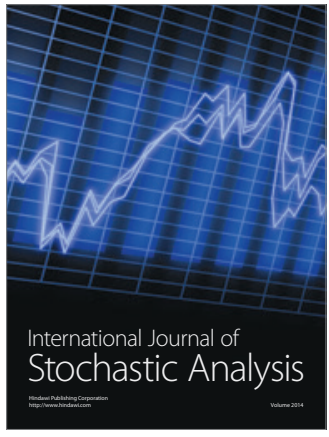

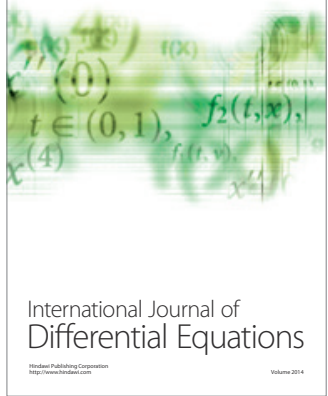
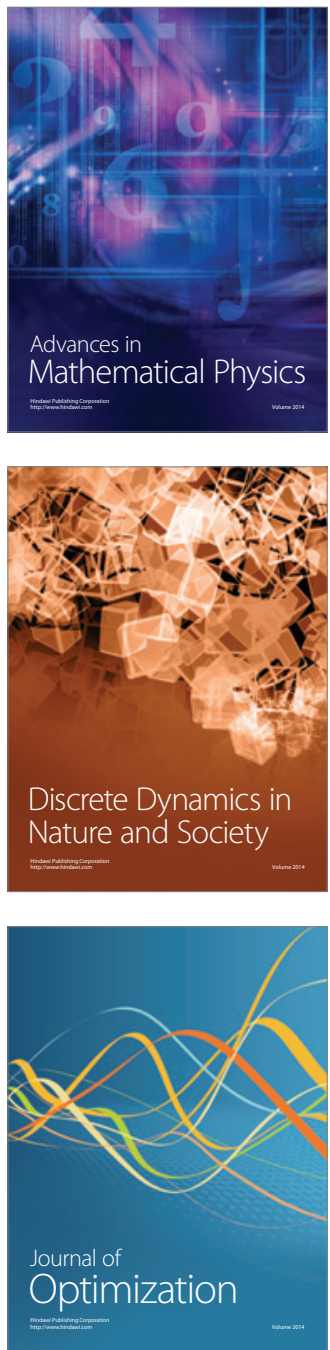